Classification

Physics Abstracts

$61.80 \mathrm{~F}$

\title{
Monte Carlo Calculation of the X-Ray Depth Distributions in an Aluminum Target
}

\author{
Khaled Raouadi $\left({ }^{1}\right)$ and Zouheir Fakhfakh $\left({ }^{2}\right)$ \\ (1) Faculté des Sciences de Tunis, Département de Physique, Tunisie \\ $\left({ }^{2}\right)$ Faculté des Sciences de Sfax, Département de Physique, Tunisie
}

(Received July 11, 1994; accepted September 20, 1995)

\begin{abstract}
A Monte-Carlo simulation of inelastic scattering process developed by Terrissol has been modified and used to calculate X-Ray depth distributions. The elastic scattering is modelled using the Mott collision cross-section and the Hartree-Fock atomic potential. The validity of this model is discussed in a comparison between the Monte-Carlo results and experimental data describing the energy distribution of backscattered electrons and energy loss data.
\end{abstract}

\section{Introduction}

Shimizu [1] and Terrissol [2] had described realistic models which simulate individual events during interaction between incident electron beam and target; they consider electron trajectories in the target as a chain of linear segments (free path) between successive interactions with the target atoms. After each interaction, the energy and the direction of the primary electron change; a secondary electron may also be created and the treatment of this electron is included in the program. A detailed description of this program and of the physics bases intervening in all the stages of calculation made the object of the recent Henoc and Maurice work [3].

We use the proposed model to calculate distribution of $\mathrm{L}$ ionizations in depth, the energy deposit profile and the energy distribution of backscattered electrons from aluminium target.

\section{Depth Distribution of $K$ and $L$ Ionizations}

For an electron of energy $E$, the kind of interaction is sampled during the run of the program. A sufficiently energetic electron can interact with an atom and cause the ejection of a tightly bound inner-shell electron, leaving the atom in an ionized and highly energetic state. Subsequent decay of this excited state result in the emission of characteristic X-Rays. Interaction producing the inner-shell ionization are computed; by calculating the number of $\mathrm{K}$ or $\mathrm{L}$ ionizations produced in a thin layer of aluminium isolated in the vacuum, we simulate the depth distribution $\varphi_{\mathrm{j}}(\rho z)$ given 
by:

$$
\varphi_{\mathrm{j}}(\rho z)=\frac{N_{\rho z}}{N_{0}}
$$

where $\mathrm{j}$ is the shell index, $N_{\rho z}$ and $N_{0}$ are the number of ionization in the imbedded layer and the isolated respectively and $\rho z$ is the mass thickness.

Henoc and Maurice [3] simulate $\varphi_{\mathrm{K}}(\rho z)$ at $20 \mathrm{keV}$ using 50000 trajectories for improving precision, they compare their results with other technics such as multiple scattering models, the single scattering one and Reimer calculations (Fig. 1).

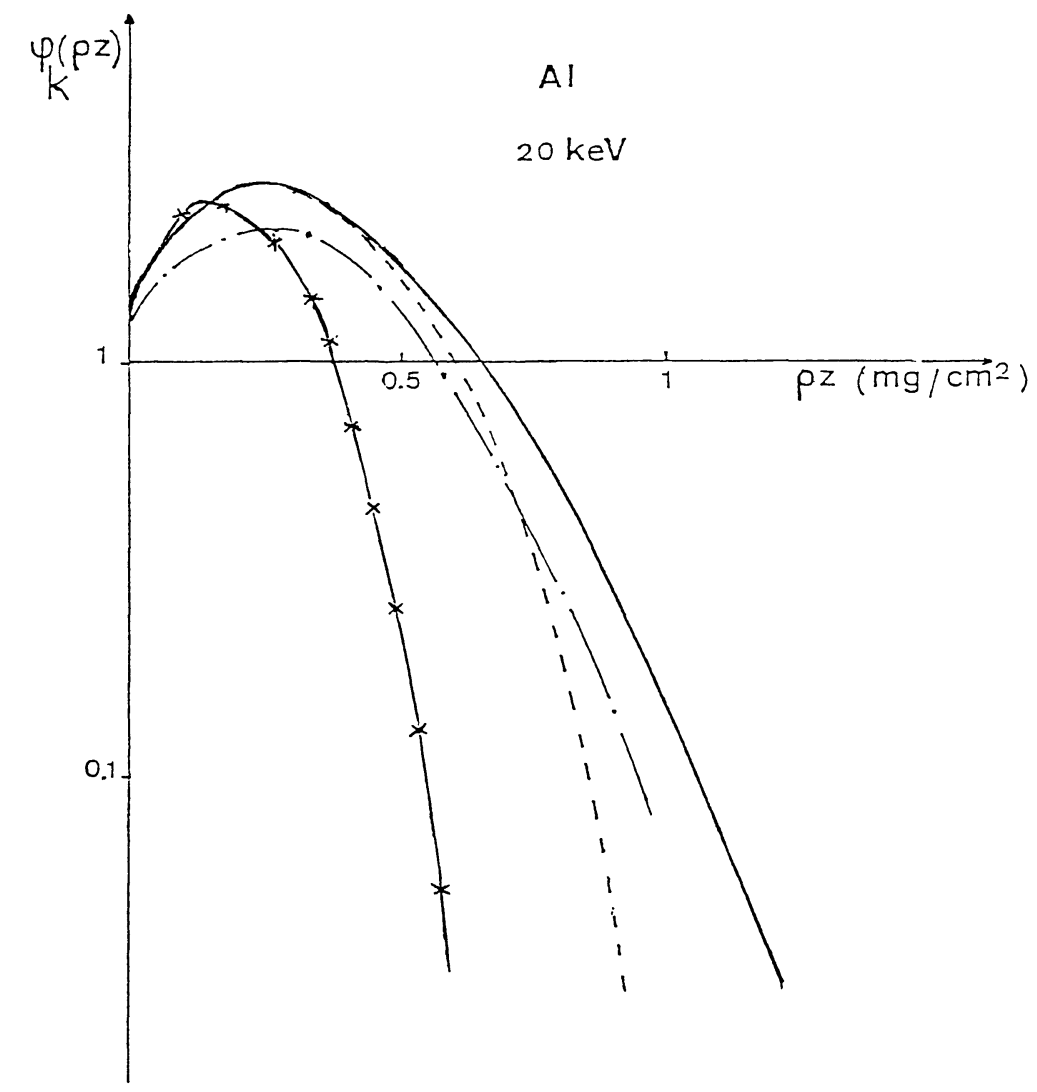

Fig. 1. - Depth distribution of Al.K-ionization at $20 \mathrm{keV}$. ( - ) Experimental (Castaing - Henoc). $(-x-)$ Terrissol model modified by (Maurice - Henoc) with Möller energy Loss. ( ---$)$ Krefling - Reimer (1976). (-- ) Single or multiple scattering model.

The curve published by Reimer [4] shows a faster decrease with increasing depth; the Terrissol treatment gives a much faster decrease; the elastic cross section is not involved: both use the Mott formula. Henoc and Maurice have substituted the Gryzinski energy loss cross section for the Möller cross section; the agreement with experimental results is not improved. They assumed that the energy loss is constant, equal to the binding energy but in this case the mean ionization depth is then greater than that observed.

Since the program gives the number of $\mathrm{K}$ and $\mathrm{L}$ ionizations and the order of magnitude of $\mathrm{L}$ ionizations in a layer $\Delta(\rho z)$ is much greater than $\mathrm{K}$ ionizations one, we introduce a new version 
of the program exploited by Henoc and Maurice. In this version we are not concerned by the Kshell excitation only; so that the description of the trajectory may be continued after the K-shell excitation.

The result of our simulation (Fig. 2) using 2500 at 5000 trajectories to calculate $\varphi(\rho z)$ is compared with the experiments of Castaing and Henoc [5].

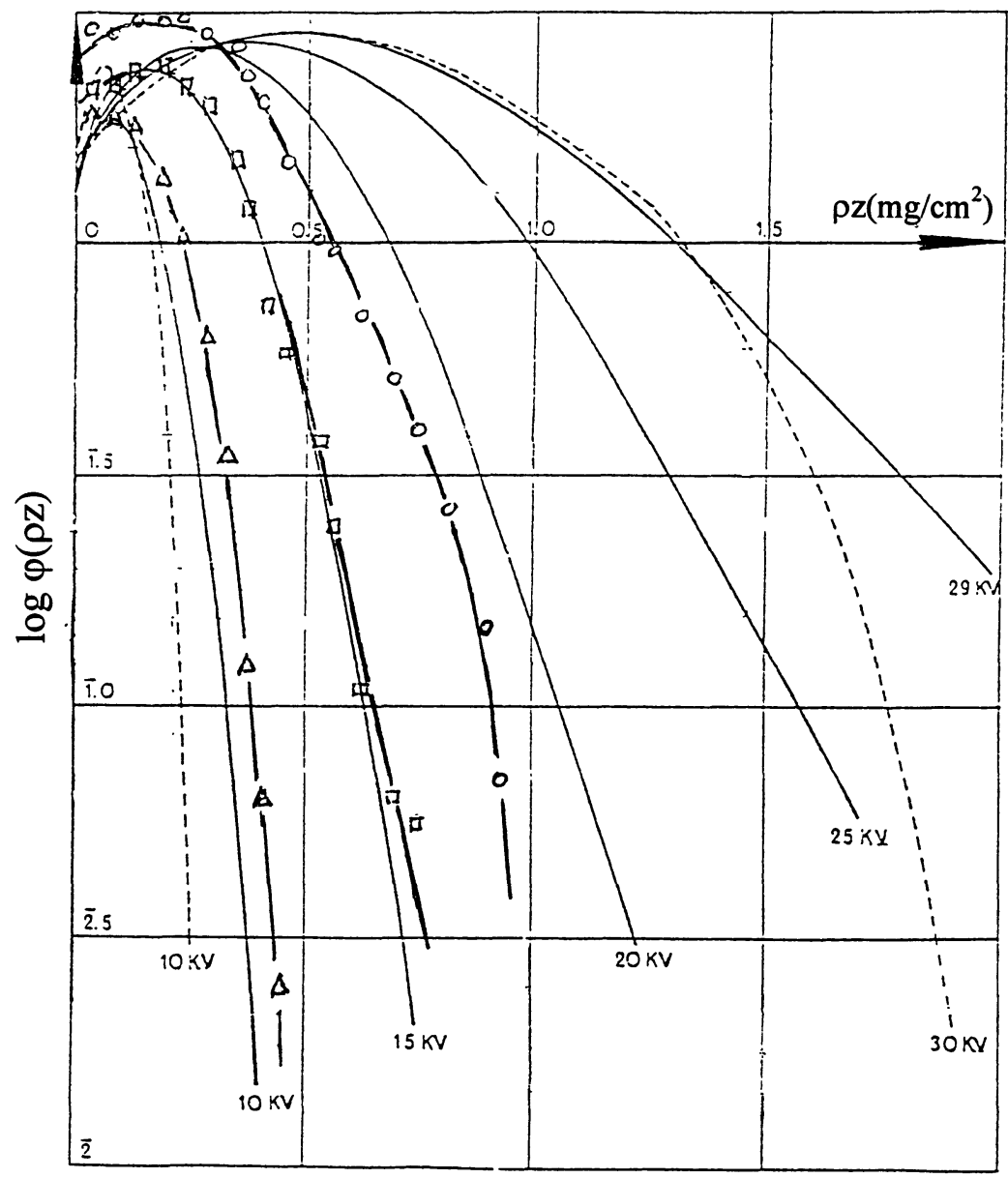

Fig. 2. - Ionization depth distribution in $\mathrm{Al}$. (-) Experimental (Castaing - Henoc) $\mathrm{Al} \mathrm{K \alpha}$. Our simulation of $\mathrm{Al} \mathrm{L \alpha}:(-\mathrm{o}-)(20 \mathrm{keV})(\square)(15 \mathrm{keV})(\triangle)(10 \mathrm{keV}) .(\ldots-)$ Bishop calculations.

The distributions of $\mathrm{K}$ and $\mathrm{L}$ ionizations ar quite similar. This can be explained as follows:

- the cross section for the L-shell ionization calculated by Shimizu [1] for an aluminum target as a function of energy (Fig. 3) is observed to decrease with increasing energy;

- for the elastic scattering cross section, the Mott formula is believed to remain accurate at low energy;

- the excitation rate remain sufficiently great for L-shell excitation with decreasing energy. 


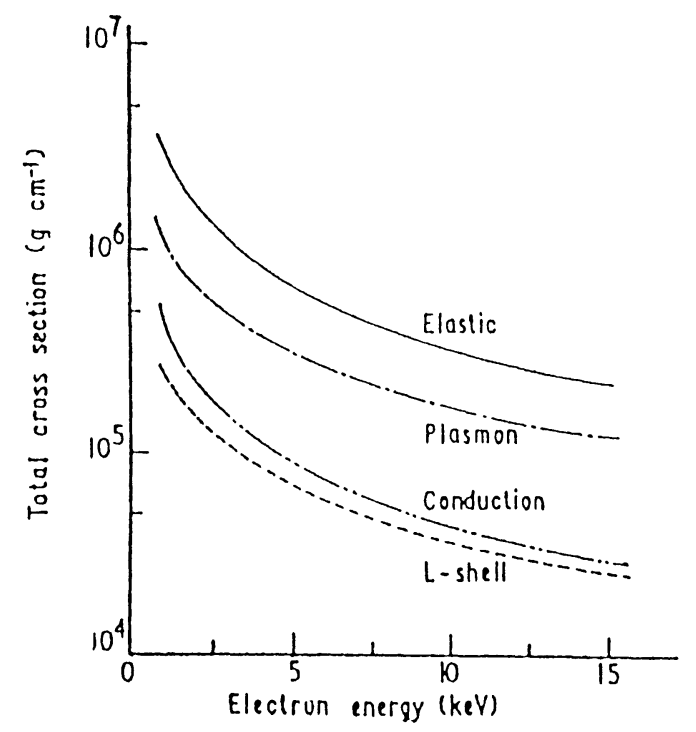

Fig. 3. - Plot of total cross section for elastic scattering and several inelastic scattering processes (Shimizu et al., 1976).

\section{Backscattering and Energy Loss}

The energy distribution of the backscattered electrons has been calculated by means of Monte Carlo techniques by several authors; example of these distribution are shown in Figure 4 along with experimental results.

Usually, the correspondence is good, although the position of the high energy peak and the shape of the distribution is somewhat dependent on the form chosen for the scattering model.

We calculate the energy distribution of backscattered electron from the Henoc-Maurice single scattering model [6] which has been modified by the authors.

In the other way, we calculate this energy distribution from the "Terrissol Model" by introducing two restrictions in the original program:

- when the primary electron energy falls below the K-shell excitation, the description of the trajectory may be ended;

- elastic cross sections are calculated using an extrapolation relation in the interval [1-20] keV.

Figure 5 shows comparison between $\mathrm{d} \eta / \mathrm{d} W$ Monte-Carlo simulated and experimental Matsukawa [7] values for aluminum at $20 \mathrm{keV}$.

The process of elastic and inelastic scattering operate concurrently. Elastic scattering causes the beam electrons to deviate from their original direction of travel, causing them to diffuse through the solid. Inelastic scattering progressively reduces the energy of the beam electron until it is captured by the solid. Thus limiting the range of travel of the electron within the solid.

Results in Figure 5 shows an increase of backscattered electron toward high energy in the Terrissol model in comparison with the single scattering model. We involve to explain this difference the elastic cross section: Mott formula seems more realistic than Rutherford description. Towards lower energy Monte carlo simulation using the single scattering model gives good concordance with the experiments; we deduce that it is useful in this case to consider all inelastic processes grouped together to be described by the continuous energy loss Bethe formula.

The major advantage to describe the cross section for the individual processes as treated in the 


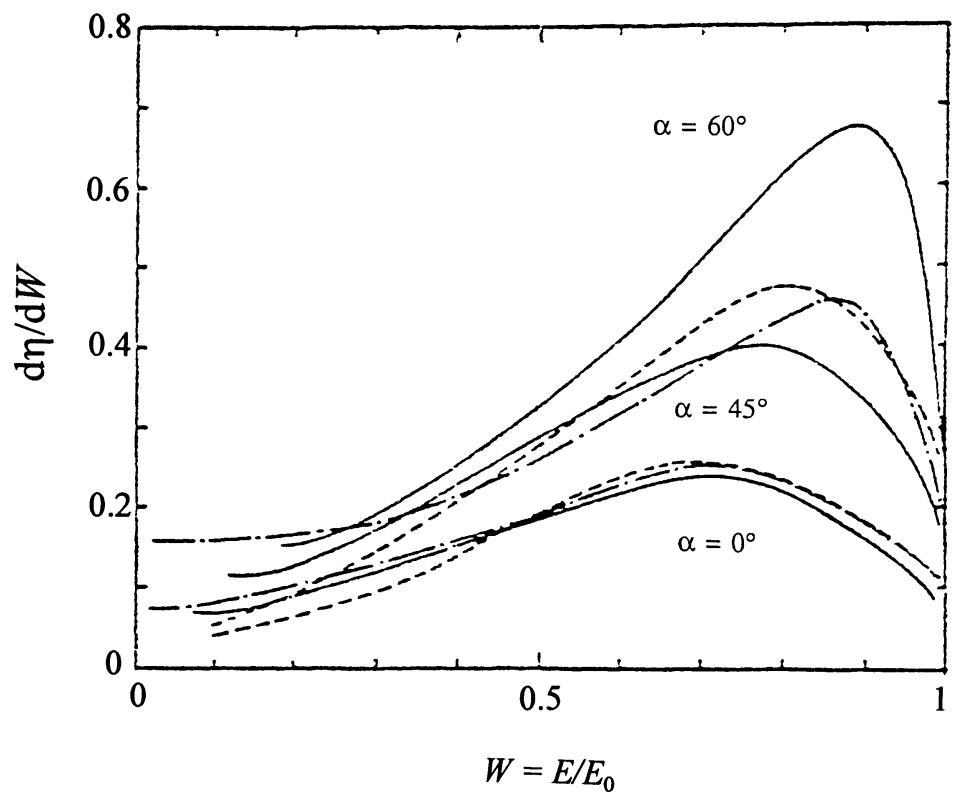

Fig. 4. - Energy spectra for $\mathrm{Al}$ at $20 \mathrm{keV}$; Full curve: experiment; broken curve: Monte Carlo; chain curve: Kulenkampff and Spyra (Matsukawa et al., 1974).

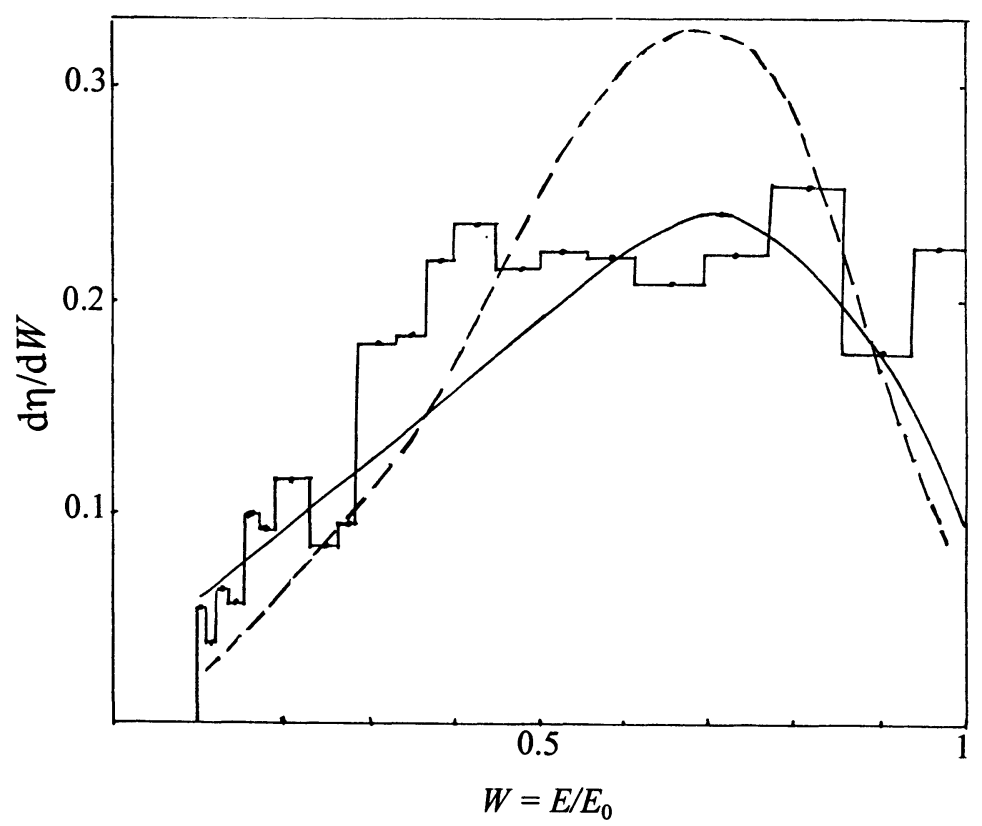

Fig. 5. - Energy spectra for $\mathrm{Al}$ at normal incidence (20 keV): Monte carlo calculations. ( - Experimental. ( - . - ) Single scattering model modified by the authors. (-- ) Terrissol model modified by the authors $(\eta=0.171)$. 


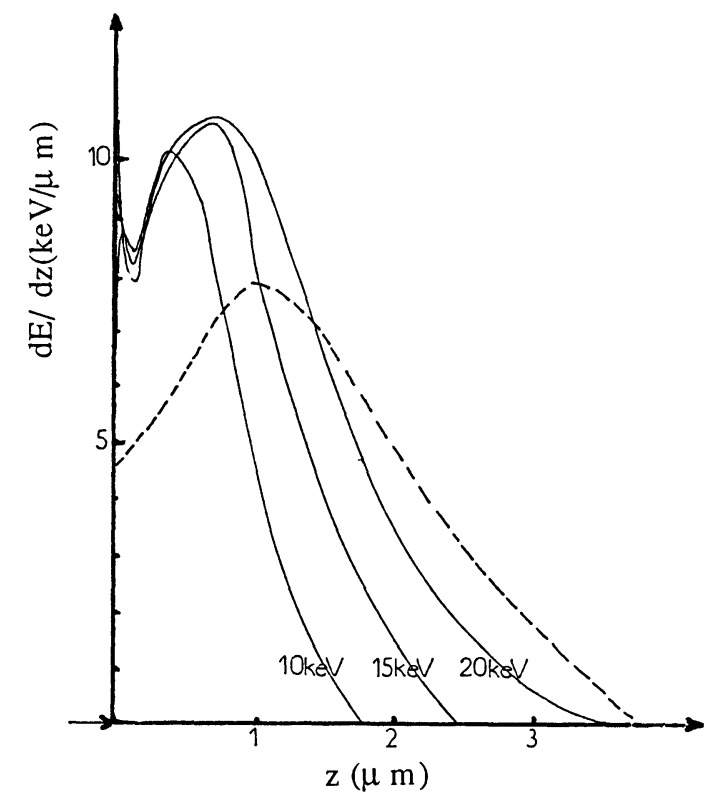

Fig. 6. - Energy deposit curves for $\mathrm{Al}$ at normal incidence. (-) Present Monte-Carlo calculations (10-15 and $20 \mathrm{keV}) .(-. .-)$ Shimizu model $(20 \mathrm{keV})$.

Terrissol model is to simulate the energy deposit.

In Figure 6, we present our simulation energy deposit results for aluminum at 20,15 and 10 $\mathrm{keV}$ and the curve obtained by Shimizu et al. [8] at $20 \mathrm{keV}$. We observe that the electron range is in a good agreement with the Bethe range at $10 \mathrm{keV}(1.8 \mu \mathrm{m})$ and that the depth of the maximum deposit is close.

\section{Conclusion}

The simulation of depth distributions of ultra soft $\mathrm{X}$-ray radiations may give some valuable idea of what can be expected in the range of energy showing the lack of experimental data. So we have considered the L-Shell ionization in aluminum and we pointed out that 2500 at 5000 trajectories are sufficient to have a good description which reduce the time process execution; the energy deposit profile provided by this Monte carlo calculation is a spring of informations about the behavior of materials under the beam irradiation, we count generalize our study in this direction.

\section{References}

[1] Shimizu R., Katokar T., Ikuta T., Hoshikawa T. and Hashimoto M., J. Phys. D (Appl. Phys) 9 (1976) 101.

[2] Terrissol M., Thesis, Université Paul Sabatier, Toulouse (1978).

[3] Henoc J. and Maurice F., Electron probe Quantitation, K.F.J. Heinrich and D.E. Eds. (Newbury, Plenum Press, New York, 1991).

[4] Reimer L. and Krefling E.R., NBS Spec publ. 460 (1976) 45.

[5] Castaing R. and Henoc J., ICXOM Orsay 1965 (Hermann Paris, 1966) p. 120.

[6] Henoc J. and Maurice F., J. Micros.Spect. Elect 5 (1980) 347.

[7] Matsukawa, Shimizu R. and Hashimoto M., J. Phys. D7 (1974) 5.

[8] Shimizu R., Ikuta T. and Murata K., J. Appl. Phys. 43 (1972) 4233. 